\title{
MANFREDA GALVANIAE (AGAVACEAE), ESPECIE NUEVA DE MÉXICO, CON NOTA SOBRE LA UBICACIÓN TAXONÓMICA DE M. MALINALTENANGENSIS MATUDA Y SU LECTOTIPIFICACIÓN
}

\author{
Alejandro Castañeda Rojas, I. Sonia Franco Martínez \\ y Abisaí García-Mendoza \\ Jardín Botánico, Instituto de Biología \\ Universidad Nacional Autónoma de México. \\ Apdo. postal 70-614 04510 México, D.F. \\ correo electrónico: abisai@ibiologia.unam.mx
}

\section{RESUMEN}

Se describe e ilustra Manfreda galvaniae, especie nueva para la ciencia, de la Barranca de Malinaltenango, mpio. de Ixtapan de la Sal, Estado de México. Esta especie pertenece al grupo de Manfreda guttata (Jacobi et Bouché) Rose y muestra similitudes con M. pringlei Rose y M. fusca Ravenna. Asimismo, se presentan comentarios acerca de la ubicación taxonómica de M. malinaltenangensis Matuda, simpátrica con M. galvaniae.

Palabras clave: Agavaceae, especie nueva de Manfreda, México.

\section{ABSTRACT}

Manfreda galvaniae, a new species from the Barranca de Malinaltenango, mpio. Ixtapan de la Sal, State of Mexico, is described and illustrated. It belongs to the Manfreda guttata (Jacobi et Bouché) Rose group, and is similar to M. pringlei Rose and M. fusca Ravenna. Likewise, the paper presents comments about the taxonomic status of $M$. malinaltenangensis Matuda, sympatric with M. galvaniae.

Key words: Agavaceae, new species of Manfreda, México.

Durante el estudio de la familia Agavaceae para el Estado de México, se encontraron plantas del género Manfreda cuyas características morfológicas difieren de las ya descritas, por lo cual se propone su ubicación en una especie nueva. 
Manfreda galvaniae A. Castañeda, S. Franco et García-Mend., sp. nov. Figs. 1, 3.

Planta herbacea, cormo globoso vel subcylindraceo, reliquiis basalibus foliorum membranaceis. Folia 22-62 cm longa, 0.6-2.6 cm lata, lineari-lanceolata, erecta, viridia, basem versus angustata, pseudopetiolum vaginantem ca. $3.0-4.0 \mathrm{~cm}$ longum $1.0 \mathrm{~cm}$ latum formantia. Flores $2.7-4.2 \mathrm{~cm}$ longi, ascendentes, semisucculenti, rubelli vel luteo-virides, ex purpureo suffusi, ovarium $0.5-0.8(-1.2) \mathrm{cm}$ longum, $0.2-$ $0.3(-0.7) \mathrm{cm}$ latum, cylindricum, $1 \mathrm{~mm}$ in tubum floralem penetrans; tubo 1.3-2.4 cm longo; segmentis 0.9-1.4 cm longis, $0.3-0.7 \mathrm{~cm}$ latis, anguste ellipticis vel ovatis, patentibus. Fructus 1.3-1.8 cm longus, 0.9-1.5 cm diametro, subglobosus.

Planta herbácea perenne, cormo 3.0-5.0 cm de largo, 2.0-4.0 cm de diámetro, subgloboso a subcilíndrico, suculento, cubierto casi en su totalidad por los restos basales de las hojas secas que miden 4.8-7.3 cm de largo, membranáceos; raíces carnosas y fibrosas. Hojas 2-5(-7), de 22-62 cm de largo, 0.6-2.6 cm de ancho, linearlanceoladas, angostándose hacia la base donde forman un pseudopecíolo de aproximadamente $3.0-4.0 \mathrm{~cm}$ de largo y $1 \mathrm{~cm}$ de ancho, semisuculentas, margen entero con una banda hialina sólo perceptible al microscopio, erectas o recurvadas, de color verde claro, glabras en ambas superficies, ápice acuminado. Inflorescencia $77-154 \mathrm{~cm}$ de alto, erecta a ligeramente arqueada, rojiza o verdosa con tintes purpúreos, pedúnculo $51-116 \mathrm{~cm}$, con $5-8$ brácteas, $2.4-55 \mathrm{~cm}$ de largo, $0.5-1.9 \mathrm{~cm}$ de ancho, las basales linear-lanceoladas, similares a las hojas, las superiores deltoides, la primera 16-55 cm de largo, (0.6-)0.9-1.9 cm de ancho, la segunda 12.1-36.3 cm de largo, 0.6-1.6 cm de ancho; porción floral 23-67 cm de largo, laxa, con 7-21 flores; brácteas florales $0.8-3.1 \mathrm{~cm}$ de largo, 0.3-0.9 cm de ancho, deltoides. Flores 2.7$4.2 \mathrm{~cm}$ de largo, ascendentes, sésiles, rojizas a verde-amarillentas con tintes purpúreos, semisuculentas, sin olor; ovario 0.5-0.8(-1.2) cm de largo, 0.2-0.3(-0.5) $\mathrm{cm}$ de ancho, cilíndrico, prolongándose $1 \mathrm{~mm}$ hacia el tubo; tubo 1.3-2.4 $\mathrm{cm}$ de largo; segmentos del perianto 0.9-1.4 cm de largo, 0.3-0.7 cm de ancho, angostamente elípticos a ovados, extendidos, verde-amarillentos con máculas purpúreas; filamentos 1.5-2.4 cm de largo, exceden al tubo por 1.0-2.0 cm, insertos a 3/4 partes del ápice del tubo; anteras $0.8-1.2 \mathrm{~cm}$ de largo, $0.1 \mathrm{~cm}$ de ancho, amarillentas; estilo 2.8-3.6 cm de largo en la antesis, blanquecino con máculas purpúreas, estigma trilobado. Frutos capsulares, $1.3-1.8 \mathrm{~cm}$ de largo, 0.9-1.5 cm de ancho, subglobosos, con marca del ápice de la cápsula, perianto persistente. Semillas $0.3-0.5 \mathrm{~cm}$ de largo, 0.2-0.4 cm de ancho, deltoides, con ala angosta, negras, brillantes.

Tipo: México, Estado de México, municipio de Ixtapan de la Sal, ladera S de la Barranca de Malinaltenango, $1450 \mathrm{~m}$, selva baja caducifolia perturbada, 14 julio 1998, A. Castañeda Rojas et al. 108 (holotipo: MEXU; isotipos: ENCB, IEB, MO). 


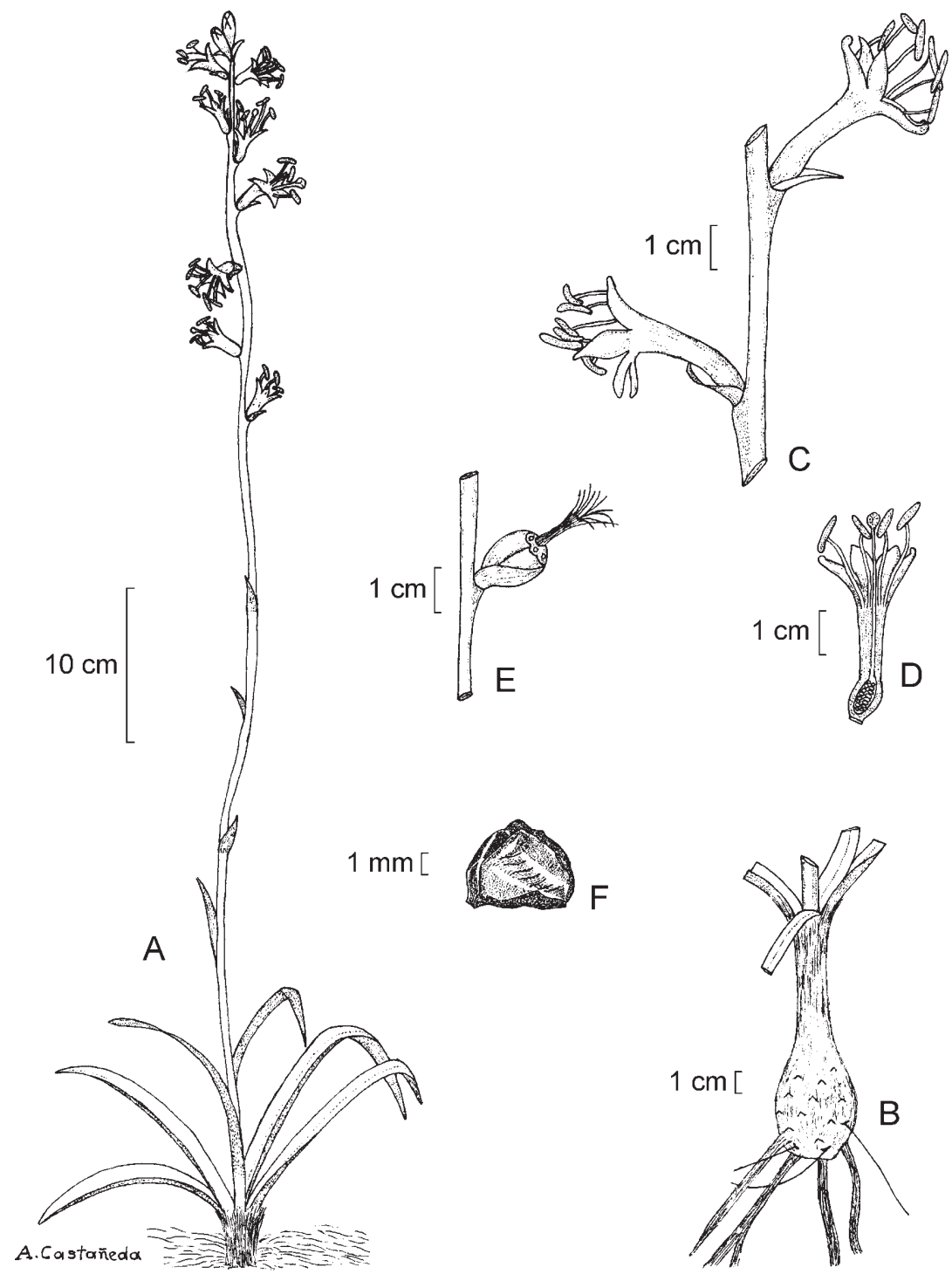

Fig. 1. Manfreda galvaniae. A. Planta con inflorescencia; B. Cormo desprovisto de los restos basales de las hojas secas; C. Flores; D. Corte longitudinal de la flor; E. Fruto; F. Semilla. (A y B basados en Castañeda Rojas et al. 108; C y D en García-Mendoza et al. 6624; E y F en Castañeda Rojas et al. 39). 
Paratipos: México, Estado de México: municipio de Ixtapan de la Sal, barranca de Malinaltenango, A. Castañeda Rojas et al. 39 (ENCB, MEXU); misma localidad, A. Castañeda Rojas 85 (MEXU); $6 \mathrm{~km}$ al S de Malinaltenango, en la barranca, A. Castañeda Rojas y A. García-Mendoza 99 (MEXU); $1 \mathrm{~km}$ al S de Malinaltenango, A. García-Mendoza et al. 6085 (MEXU); Promontorio rocoso camino de Malinaltenango hacia el río, A. García-Mendoza et al. 6624 (MEXU, MO); municipio de Malinalco, zona arqueológica de Malinalco, A. Castañeda Rojas 93 (MEXU); municipio de Temascaltepec, $\mathrm{km} 4$ al SO de Temascaltepec, camino a San Pedro Tenayac, A. Castañeda Rojas et al. 47 (ENCB, MEXU); municipio de Ocuilan, km 39 de la carretera a Chalma, A. Castañeda Rojas 87 (MEXU); misma localidad, A. Castañeda Rojas 137 (MEXU).

Distribución y hábitat: Manfreda galvaniae crece en altitudes de 1450-1900 m s.n.m. Forma parte de la selva baja caducifolia y su ecotonía con el bosque de Quercus, donde se asocia con Arracacia, Commelina, Echeandia, Jatropha, Opuntia, Tigridia y Zephyranthes. Florece en el mes de julio y fructifica en agosto y septiembre. Aparentemente es endémica del Estado de México donde se distribuye en los municipios de Ixtapan de la Sal, Malinalco, Ocuilan y Temascaltepec. El nombre de esta especie se dedica a la Dra. Raquel Galván Villanueva del Instituto Politécnico Nacional, especialista en la familia Agavaceae.

Discusión: Manfreda galvaniae pertenece al grupo de M. guttata, reconocido por Verhoek-W. (1975) en función de sus características foliares y florales, hojas delgadas a semisuculentas con margen hialino, liso o áspero al tacto y entonces papiloso o eroso-denticulado al microscopio, ovario proyectado ligeramente hacia el interior del tubo floral, perianto persistente en las cápsulas, estas últimas con una cicatriz anuliforme alrededor del ápice. Al grupo pertenecen Manfreda fusca Ravenna, M. guttata (Jacobi et Bouché) Rose, M. littoralis García-Mend., Castañeda y Franco, M. planifolia (S. Watson) Rose, M. pringlei Rose y M. rubescens Rose (García-Mendoza et al., 2000).

Manfreda galvaniae se distingue por su cormo globoso, casi siempre cubierto en su totalidad por los restos basales membranáceos de las hojas secas; hojas linearlanceoladas, pseudopecioladas, semisuculentas, de color verde claro; inflorescencia corta y laxa; flores semisuculentas, rojizas a verde-amarillentas con tintes purpúreos, segmentos del perianto extendidos, angostamente elípticos a ovados y ovario que se proyecta hacia el interior del tubo.

Dentro del grupo, las especies más cercanas morfológicamente a Manfreda galvaniae son $M$. fusca y $M$. pringlei, de las que se separa claramente por el margen hialino de las hojas, la disposición de las flores en la inflorescencia, la 
suculencia y color de las flores, la forma y orientación de los segmentos y el tamaño reducido de los filamentos, además de otras características mostradas en el Cuadro 1. Las especies del grupo se separan de la siguiente manera:

Clave de las especies del grupo Manfreda guttata

1 Hojas elípticas, elíptico-lanceoladas u oblongas.

2 Flores 1.5-2.0(-2.5) $\mathrm{cm}$ de largo; tubo 1.0-2.0(-2.8) $\mathrm{mm}$ de largo; los filamentos exceden al tubo por (1.0-)1.4-1.8 cm, adnados en toda la longitud del tubo M. littoralis

2 Flores 3.2-4.0 cm de largo; tubo 5.0-7.0 mm de largo; los filamentos exceden al tubo por $2.0-5.2 \mathrm{~cm}$, insertos a la mitad del tubo M. planifolia

1 Hojas lineares, linear-lanceoladas a lanceoladas.

3 Filamentos adnados en toda la longitud del tubo.

4 Hojas 0.6-1.0 cm de ancho; estambres 1.6-2.3 $\mathrm{cm}$ de largo

M. rubescens

4 Hojas (0.9-)1.3-3.1 cm de ancho; estambres (2.0-)3.0-4.1 cm de largo

M. guttata

3 Filamentos insertos a la mitad o a tres cuartas partes de la longitud del tubo.

5 Porción floral laxa; filamentos $1.5-2.4 \mathrm{~cm}$ de largo M. galvaniae

5 Porción floral congesta; filamentos 3.5-4.9 cm de largo.

6 Ovario 1.2-1.7 cm de largo; segmentos del perianto 1.5-1.6 cm de largo; especie conocida sólo de las montañas del centro de Guatemala M. fusca

6 Ovario 0.9-1.1(-1.2) cm de largo; segmentos 0.8-1.3(-1.6) $\mathrm{cm}$ de largo; especie distribuida en el Eje Neovolcánico y montañas de Oaxaca, México

M. pringlei

\section{MANFREDA MALINALTENANGENSIS MATUDA Y M. GALVANIAE}

Manfreda malinaltenangensis Matuda (1976) fue descrita con la siguiente información: "Tipo: México. Estado de México; Barranca de Malinaltenango, a 1400 m s.n.m., 5 de diciembre de 1975. Matuda 38694. (MEXU)". Acompaña a la descripción una fotografía de la inflorescencia con 7 flores senescentes. Sin embargo, al estudiar la especie, se encontraron dos problemas, uno referente a la tipificación y el segundo con su ubicación taxonómica y su probable parentesco con M. galvaniae.

La publicación señala que el holotipo se depositó en el herbario MEXU; no obstante, el número Matuda 38694, no fue localizado en esta institución, ni en una 


\begin{tabular}{|c|c|c|c|c|c|c|c|c|c|}
\hline 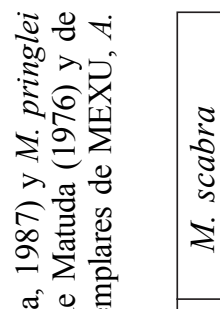 & 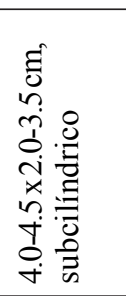 & $\left|\begin{array}{c}\hat{d} \\
\hat{d} \\
\hat{b}\end{array}\right|$ & 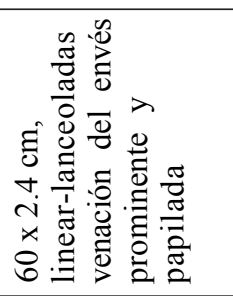 & 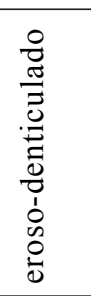 & 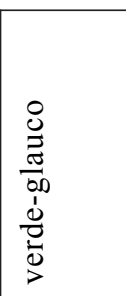 & 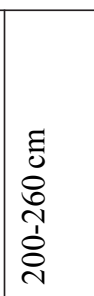 & 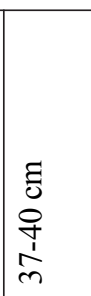 & 㞼 & m \\
\hline 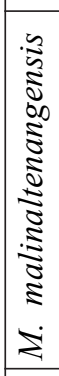 & 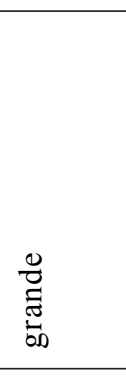 & $\frac{0}{\dot{a}}$ & 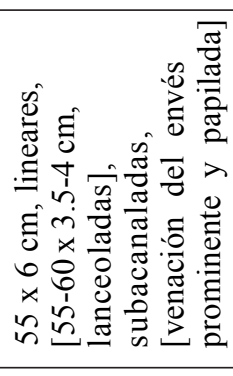 & 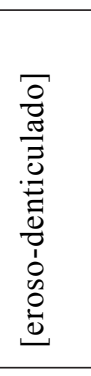 & 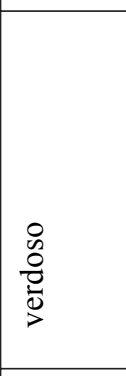 & 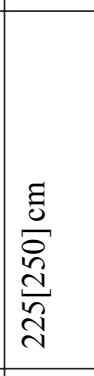 & & 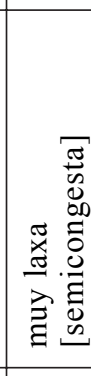 & $\underset{1}{\infty}$ \\
\hline 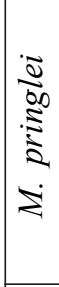 & 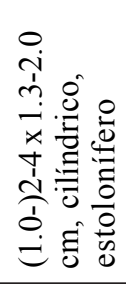 & $\mid \begin{array}{l}0 \\
\frac{1}{0} \\
\frac{1}{d} \\
\end{array}$ & 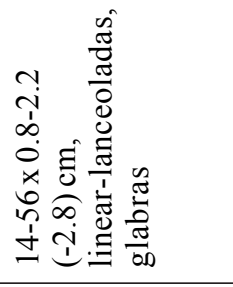 & 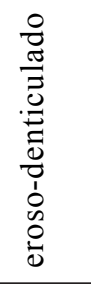 & 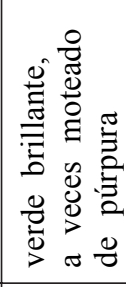 & 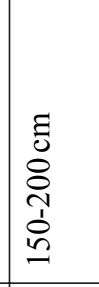 & 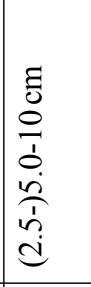 & 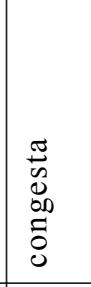 & 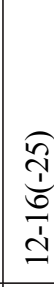 \\
\hline 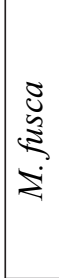 & 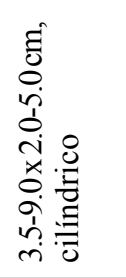 & $\simeq$ & 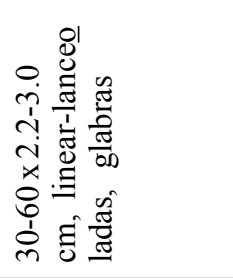 & 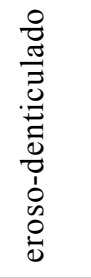 & 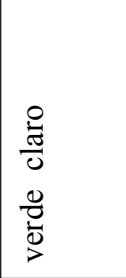 & 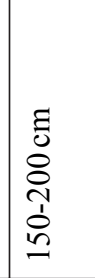 & $\begin{array}{l}\Xi \\
\varrho \\
\varrho\end{array}$ & 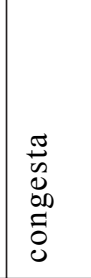 & i \\
\hline 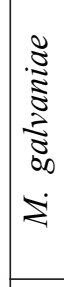 & 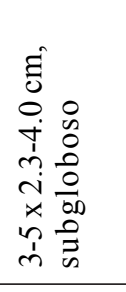 & $\left|\begin{array}{c}\hat{T} \\
\mathfrak{w} \\
\dot{d}\end{array}\right|$ & 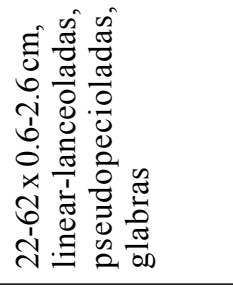 & 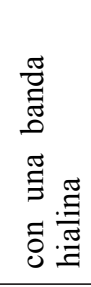 & 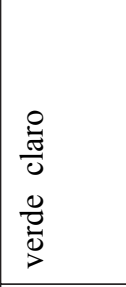 & 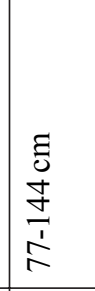 & 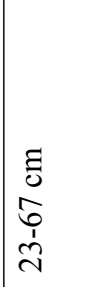 & 㞼 & 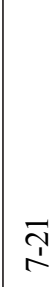 \\
\hline & 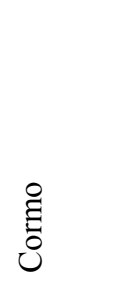 & 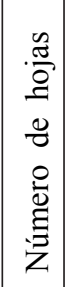 & 量营 & 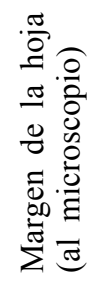 & 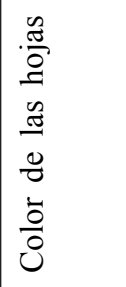 & 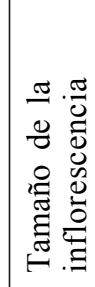 & 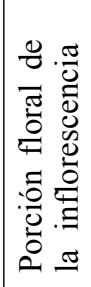 & 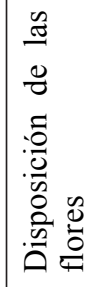 & 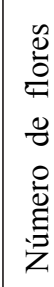 \\
\hline
\end{tabular}


Castañeda et al.: Manfreda galvaniae (Agavaceae), especie nueva de México

\begin{tabular}{|c|c|c|c|c|c|c|c|c|}
\hline 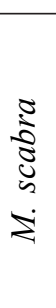 & 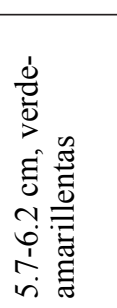 & 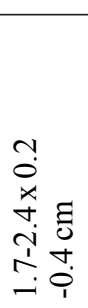 & 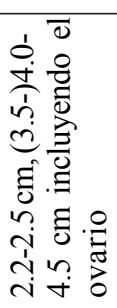 & 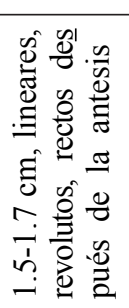 & 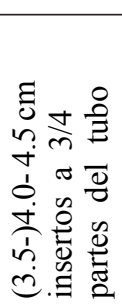 & 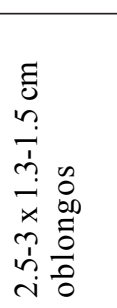 & 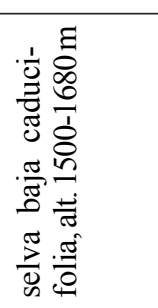 & 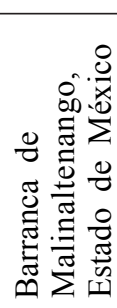 \\
\hline 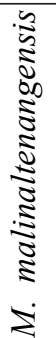 & $\begin{array}{c}\tilde{E} \\
0 \\
0 \\
0 \\
\\
\dot{1} \\
b \\
\dot{0} \\
\dot{0}\end{array}$ & 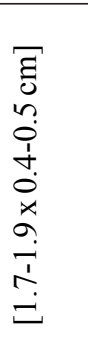 & 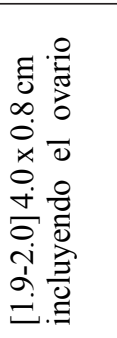 & 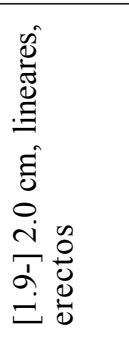 & 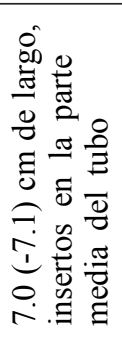 & & 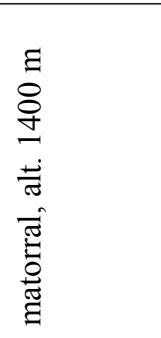 & 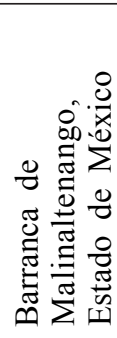 \\
\hline 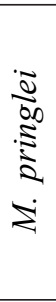 & 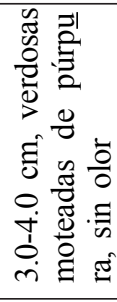 & 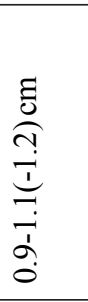 & 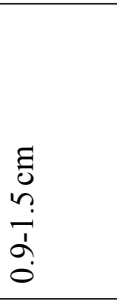 & 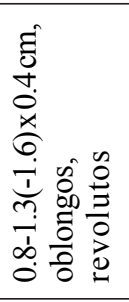 & 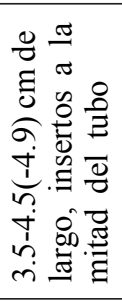 & 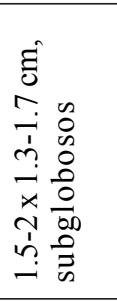 & 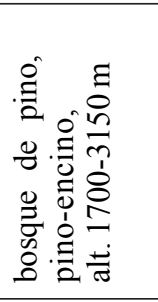 & 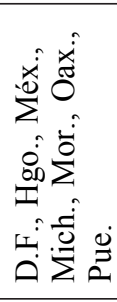 \\
\hline 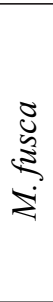 & 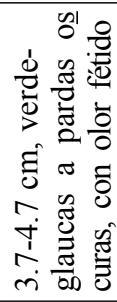 & 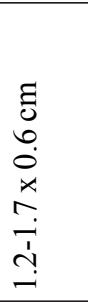 & $\begin{array}{l}\text { ठี } \\
\text { サ. }\end{array}$ & 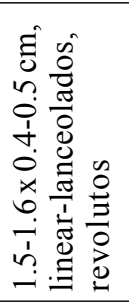 & 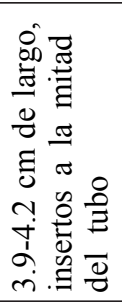 & 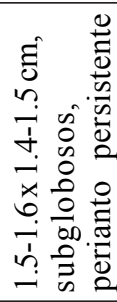 & 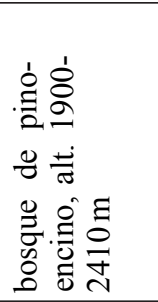 & 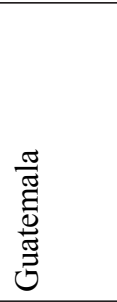 \\
\hline 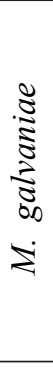 & 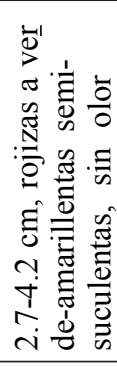 & 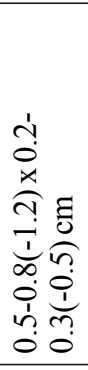 & 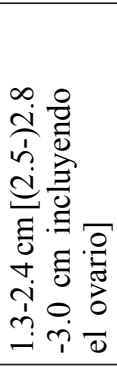 & 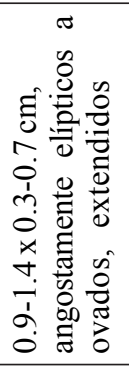 & 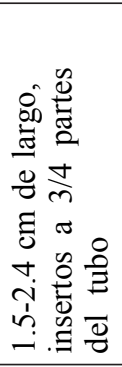 & 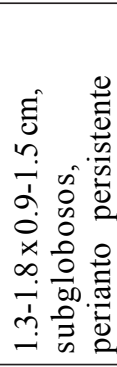 & 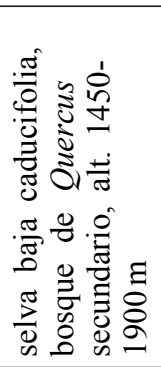 & 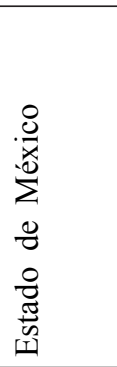 \\
\hline & $\frac{0}{0}$ & $\overbrace{0}^{0}$ & 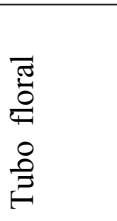 & 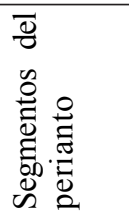 & 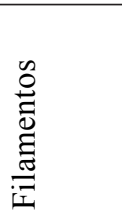 & $\underset{\stackrel{n}{0}}{\stackrel{0}{\Xi}}$ & 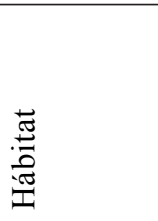 & 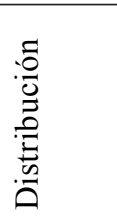 \\
\hline
\end{tabular}


búsqueda anterior hecha por Galicia (1992), quien supone que el ejemplar se extravió en el correo (en un probable envío) y que se carece de isotipos. En una segunda indagatoria se localizaron dos ejemplares de su propia colección relacionados con el nombre de la especie, pero no mencionados en el protólogo, uno de ellos en el Herbario Eizi Matuda (CODAGEM), de la Universidad Autónoma del Estado de México, y el segundo en el Herbario Nacional MEXU. Ambos ejemplares comparten la siguiente información: "Manfreda malinaltenanguensis Matuda Sp. nov. Barranca de Malinaltenango, Tonatico, México. Noviembre 15 de 1975. Matuda 38695", tienen además una fotografía posiblemente de la misma inflorescencia del ejemplar 38694, pero al inicio de la floración.

El espécimen de la CODAGEM, es el más completo, ya que posee una hoja, dos flores, una fotografía y proporciona información de la planta y el tipo de vegetación. El ejemplar de MEXU (285804) es una mezcla de dos géneros, la hoja corresponde a Manfreda, y tiene una ramilla con 9 flores pertenecientes a Furcraea pubescens Tod. Las medidas morfológicas de las hojas y las flores corresponden a aquellas de la descripción original de M. malinaltenangensis. La única discrepancia entre el espécimen citado como holotipo y estos dos ejemplares es el número de colecta anterior del holotipo. Galicia (1992) atribuye las inconsistencias en algunas colectas del autor, a que Eizi Matuda no organizó sus datos en una libreta de campo, y en su lugar existe un fichero (depositado en MEXU) que no tiene información más amplia que la que proporciona cada etiqueta, lo que dificultó el manejo de la información y provocó que hubiera diferencias en su numeración y fechas, e incluso localidades. Así, parece que Matuda hizo dos colectas de la especie, la primera el 15 de noviembre y la segunda el 5 de diciembre, y de ambas tomó fotografías.

Es indudable que estos dos especímenes localizados son parte del material original visto y utilizado por el autor para hacer la descripción que valida el nombre, por lo que de acuerdo con el artículo 9.2 del Código Internacional de Nomenclatura Botánica (Greuter et al., 2002), ante la pérdida del holotipo, designamos un lectotipo.

Manfreda malinaltenangensis Matuda, Cact. Suc. Mex. 21:74, fig. 28. 1976. LECTOTIPO, aquí designado: MÉXICO. Estado de México: (municipio de Ixtapan de la Sal), barranca de Malinaltenango, Tonatico, 15 noviembre 1975, E. Matuda 38695 (CODAGEM!, Isolectotipo MEXU! 285804, pro parte). Fig. 2.

La localización de estos dos ejemplares permite ahora ubicar taxonómicamente a $M$. malinaltenangensis dentro del género, situación que con anterioridad había sido difícil (S. Verhoek-Williams, com. pers.). Durante diferentes temporadas de los años 1994-1998, los autores visitaron la barranca de Malinaltenango y en esos recorridos se observaron dos especies simpátricas que fueron recolectadas, 


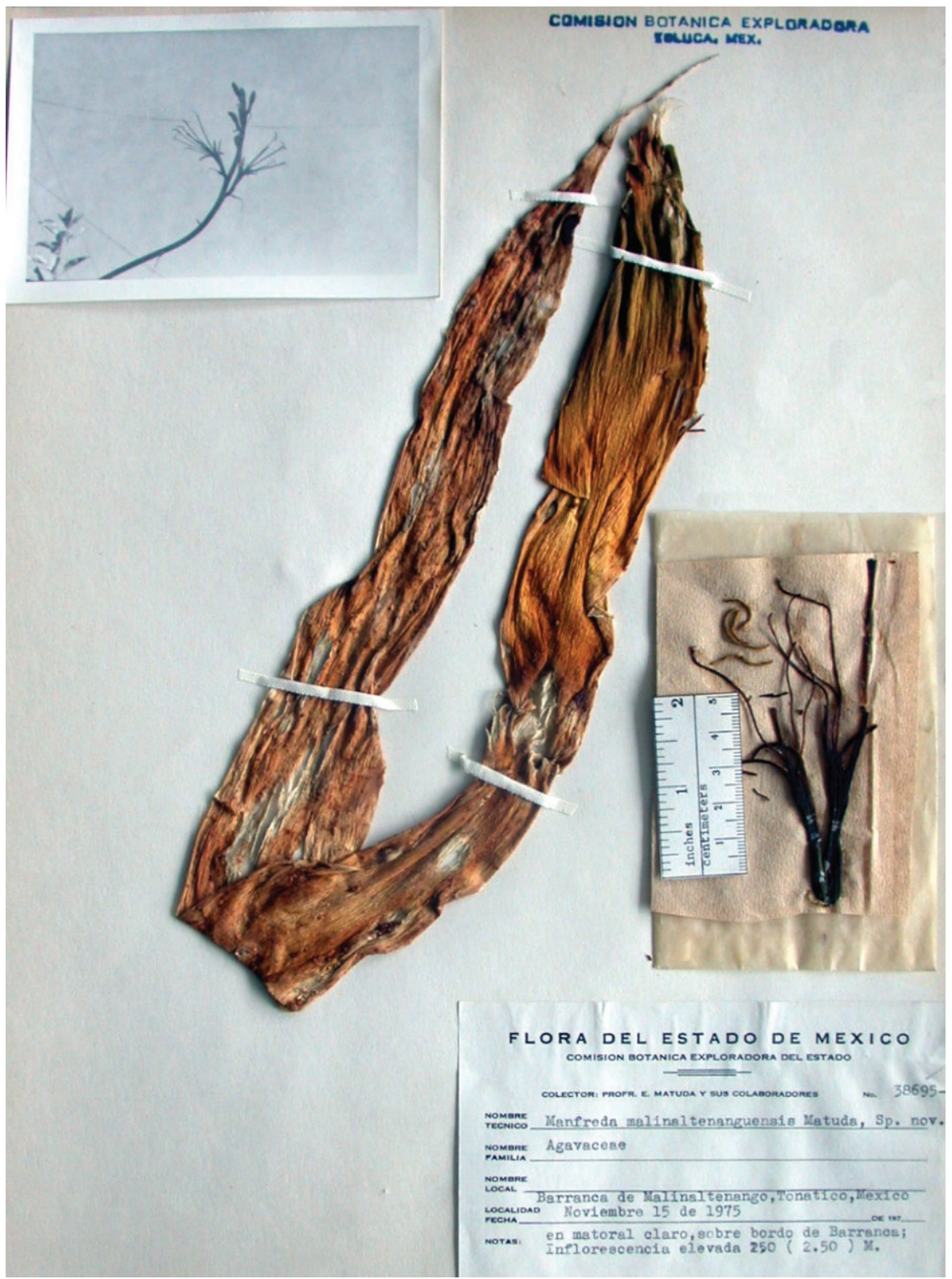

Fig. 2. Manfreda malinaltenangensis Matuda. Tipo: México. Estado de México, (municipio de Ixtapan de la Sal), barranca de Malinaltenango, 15 noviembre 1975, E. Matuda 38695 (lectotipo: CODAGEM). 
estudiadas y comparadas con la información del protólogo de M. malinaltenangensis, cuya información fue corroborada y complementada con los especímenes de herbario ya citados.

En la descripción, Matuda menciona algunas características morfológicas que permiten establecer una comparación con las especies recolectadas, las más importantes son: "Hojas radicales arrosetadas, 8 a 10, con lámina entera, algo carnosa, de $55 \mathrm{~cm}$ de longitud por $6 \mathrm{~cm}$ de ancho,"... (hojas del ejemplar Matuda 38695,60 x $3.5 \mathrm{~cm}$ en la parte media, margen eroso-denticulado). "Escapo terminal, con la inflorescencia de $2.25 \mathrm{~m}$, cilíndrico, glabro, verdoso; espiga simple muy laxa; flores 7 a 8 ; ... Flores sésiles; perianto campanulado; tubo subcilíndrico, incluyendo el ovario de $4 \mathrm{~cm}$ de longitud por $8 \mathrm{~mm}$ de grosor; lóbulos del perianto lineares, gruesos, erectos, extendidos, de $2 \mathrm{~cm}$ de longitud; estambres y filamentos filiformes, naciendo de la parte media de la pared interior del tubo, de $7 \mathrm{~cm}$ de longitud, subpurpúreos y subiguales".

De la fotografía de la inflorescencia en la publicación, se infiere que la planta se encuentra en la última etapa de la floración, ya que la orientación erecta de los tépalos se manifiesta después de la antesis. Las hojas semisuculentas, el margen papilado o eroso-denticulado y la venación del envés papilada, permiten ubicarla en el grupo (y en la especie) de Manfreda brachystachya (Cav.) Rose, propuesto por Verhoek-W. (1975). Esta especie, ahora conocida como M. scabra (Ortega) McVaugh, es de amplia distribución en el país y en el estado de México se conoce de 17 municipios.

Además de las hojas, el tamaño de las flores, la longitud del ovario-tubo de la flor y los tépalos lineares, erectos (después de la antesis), son similares en $M$. malinaltenangensis y las plantas recolectadas en la barranca y determinadas como M. scabra (AC-9, AG-5892, AG-6084, en MEXU). Asimismo, M. malinaltenangensis fue recolectada hacia el fin de año, cuando $M$. scabra aún se encuentra en floración (en el Estado de México, la hemos recolectado con flores en octubre (AG-6324, Malinalco) y diciembre (AG-6130, Temascaltepec)). Con base en las características morfológicas y fenológicas, afirmamos que $M$. malinaltenangensis es un sinónimo de M. scabra. En la Figura 3 se comparan los caracteres vegetativos y reproductivos aquí discutidos.

De esta manera, Manfreda galvaniae aunque es simpátrica con M. scabra, se diferencia de ella por el tamaño de las hojas, el tipo de margen y venación, tamaño, color y suculencia de las flores, forma de los tépalos, longitud de los filamentos y marca del ápice de la cápsula. Las diferencias entre las dos especies se ponen de manifiesto en el Cuadro 1 y se observan en la Figura 3. En adición Manfreda galvaniae florece en julio, mientras que M. scabra lo hace de agosto a diciembre. 

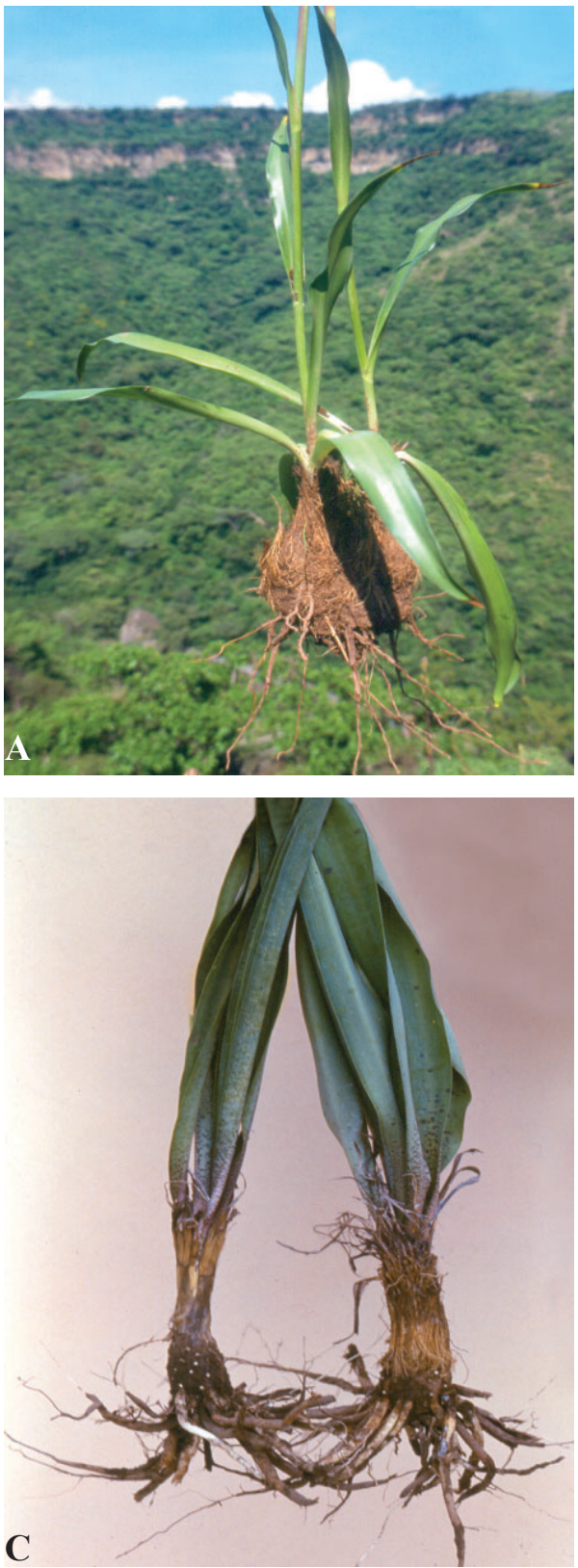
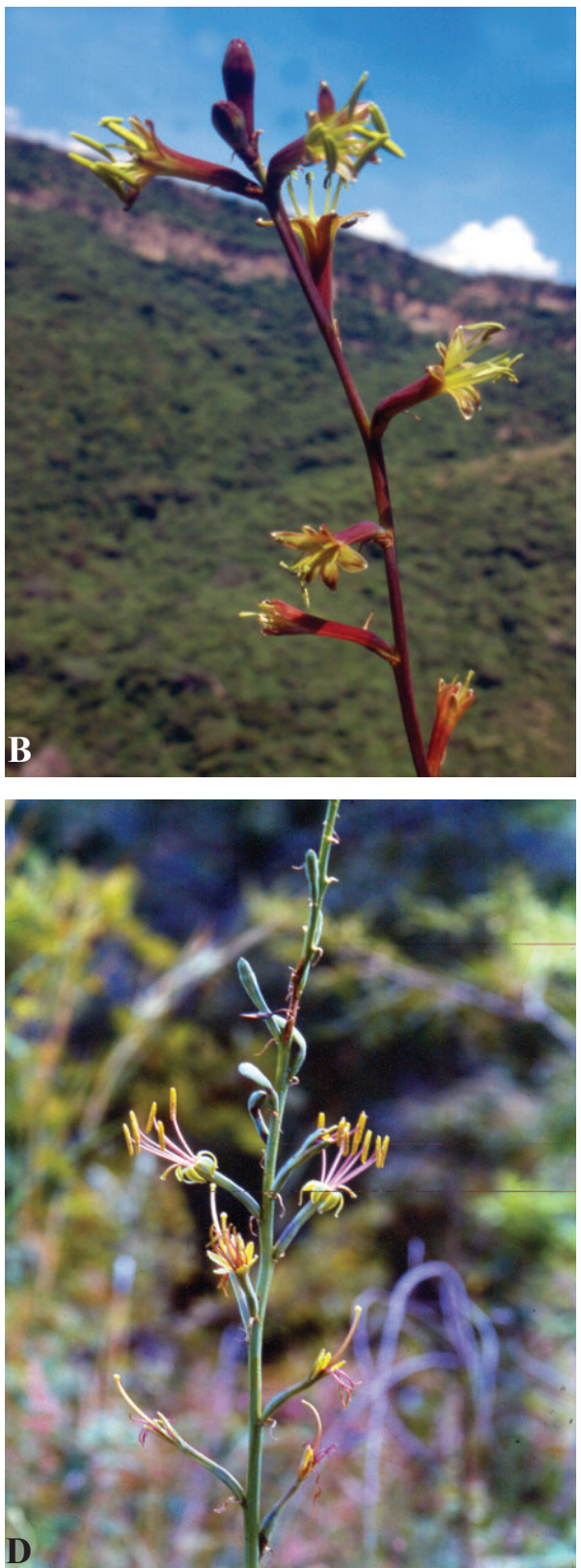

Fig. 3. A y B. Manfreda galvaniae: A. Cormos y hojas; B. Inflorescencia. C. Cormos de M. scabra (izquierda) y M. galvaniae (derecha); D. Inflorescencia de M. scabra. (A y B, del número AG-6624; C izquierda y D, del número AG-6084; C derecha, del número AG6085. Todas las fotos de la barranca de Malinaltenango. 


\section{AGRADECIMIENTOS}

Agradecemos al Dr. Fernando Chiang la diagnosis en latín y sus atinados comentarios al manuscrito; a los Drs. Jerzy Rzedowski y Eloy Solano quienes revisaron también el trabajo; al Ing. José Antonio López Sandoval, jefe del Herbario Eizi Matuda, de la Universidad Autónoma del Estado de México, por ayudarnos en la consulta de los especímenes de la CODAGEM; a Susan Verhoek-Williams, quien comentó con nosotros algunos problemas taxonómicos de la especie; asimismo a las Bióls. María del Rosario García Peña por facilitarnos el acceso al ejemplar de Manfreda malinaltenangensis, depositado en MEXU y a Teresa Germán por compartir su experiencia con las colecciones de Eizi Matuda.

\section{LITERATURA CITADA}

Galicia, M. V. 1992. Listado florístico del Estado de México y regiones circundantes (de los estados de Hidalgo, Querétaro y Distrito Federal) basado en las colecciones de Eizi Matuda. Tesis de licenciatura. Facultad de Ciencias, Universidad Nacional Autónoma de México. México, D.F. 207 pp.

García-Mendoza A., A. Castañeda e I. S. Franco. 2000. Manfreda littoralis (Agavaceae), nueva especie de Guerrero y Oaxaca, México. Acta Bot. Mex. 50: 39-45.

Greuter, W., J. McNeill, F. R. Barrie, H. M. Burdet, V. Demoulin, T. S. Filgeiras, D. H. Nicolson, P. C. Silva y J. E. Skog. 2002. Código Internacional de Nomenclatura Botánica (Código de Saint Louis). Edición en español a cargo de R. Kiesling. Instituto de Botánica Darwinion y Missouri Botanical Garden Press, St. Louis. 181 pp.

Matuda, E. 1976. Tres nuevas especies de plantas mexicanas. Cact. Suc. Mex. 21: 74-76.

Ravenna, P. 1987. Notes on Agavaceae I. A new species of Manfreda. Herbertia 43: 1719.

Verhoek-W., S.E. 1975. A study of the tribe Poliantheae (including Manfreda) and revisions of Manfreda and Prochnyanthes (Agavaceae). Tesis doctoral. Cornell University, Ithaca, Nueva York. 405 pp. 